\title{
Isolation and Molecular Charcteristic of a \\ Recombinant Feline Calicivirus from Qingdao, China
}

\author{
Yongxiang Liu ( $\nabla$ liuyongxiang0726@foxmail.com ) \\ Hebei University of Engineering https://orcid.org/0000-0003-2490-0807 \\ Xiaoliang Hu \\ Yibin University \\ Lide Qin \\ China Animal Health and Epidemiology Center
}

\section{Research Article}

Keywords: FCV, isolation, recombination

Posted Date: July 22nd, 2021

DOI: https://doi.org/10.21203/rs.3.rs-729125/v1

License: (c) (i) This work is licensed under a Creative Commons Attribution 4.0 International License.

Read Full License 


\section{Abstract}

Feline calicivirus (FCV) is a highly contagious pathogen that can cause seriously upper respiratory tract and oral disease in feline. Despite widespread vaccination, the prevalence of FCV remains high. In this study, the FCV qd/2019/china was isolated from domestic feline oropharyngeal swab which was collected in qingdao, China. The virus was purified with plaque assay and identified with PCR and IFA method. the capsid amino acid ,VP1, of qd/2019/china [1] showed sequence identity with other isolate ranging from $83.9 \%$ (ym3/2001/jp) to $91.1 \%$ (CH-JL4) .Sequence analysis of the capsid amino acid revealed that qd/2019/china was closely related to CH-JL4 and clustered with CH-JL4 in the phylogenetic tree. Phylogenetic analysis indicated that complete genome of qd/2019/china and CH-JL4 was also classified into the same cluster. While the recombination analysis with Simplot indicate that the $\mathrm{qd} / 2019 /$ china originated from the recombination of CH-JL4 and HRB-SS, and the region 3821-5301 nt originated from HRB-SS. Further, the region 3821-5301 nt belong to protease -polymerase (PP) of HRBSS. Here we isolate a new FCV qd/2019/china, which may be a recombinant virus, These results were beneficial for understanding the evolution of FCV.

\section{Importance}

Although many FCV isolates have been widely reported in China in recent years, the numbers of China FCV isolate is still not enough, the discovery of new FCV isolate could enrich the information of China strain. Here the isolation and sequence analysis of FCV qd/2019/china provids new evidence for the recombination and evolution of FCV.

\section{Introduction}

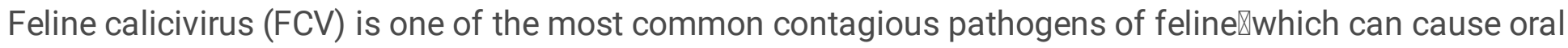
ulcer,ocular conjunctivitis and infectious upper respiratory tract disease[1] $\mathrm{FCV}$ can also infect other members of the felidae, like tigers, cheetahs[2] and lion[3] खbesides $\triangle F C V$ has also been isolated from dog faeces [4-6] . Traditionally, FCV infections are usually associated with acute, mild and self-limiting disease[7], it will not cause much harm $\mathbb{\text { If }}$ the secondary infection is well controlled.

FCV belongs to the genus Vesivirus of family Caliciviridae, [8]. It contain many virus that cannot be readily cultured on cells $₫$ such as Norovirus, Sapovirus and Rabbit hemorrhagic disease virus $\llbracket a n d$ which limit the pathogenic study of Caliciviridae. While FCV can be readily cultured on feline passaged cell lines and cause obvious cytopathic effect (CPE), then FCV has become a well in vitro model for Caliciviridae research.

FCV is single-stranded positive-sense RNA viruses $囚 i t s$ genome is approximately $7.8 \mathrm{~kb}$ in length, which covered by a capsid protein but without envelope [9],its icosahedral capsid diameter is about 27-40 nm. Due to lower fidelity, the genome of FCV bearing high mutatant rate and underwent rapid evolution, Immune system pressure may be one of the most important reasons of mutation power source邓which 
has contributed to its enhanced virulence and vaccination failure [10], One another reason may be the low fidelity of protease and polymerase(PP), PP is the RNA-dependent RNA polymerase (RdRp) of FCV, Although FCV has only one serotype, the strains isolated in different countries, regions and periods are diverse. This need further investigation.

As we all know, random genetic drift, mutation and recombination are the most common ways of viral evolution and variation, a common characteristic of RNA viruses is the high plasticity of the RNA genome, which has a high mutation rate during replication because of the low fidelity, lack of proofreading and post-replicative repair activities of the viral RNA polymerase [11] \many virus have been reported about the recombination events like Canine enteric coronaviruses (CCoVs) $[12,13]$ 『Porcine reproductive and respiratory syndrome virus (PRRSV) $[14,15]$ Influenza virus [16] \but few studies about the recombination of FCV has been reported.

However, in recent years, due to the high degree of FCV variability, there have appeared highly lethal strains, which seriously threaten the health of cats [17] . Occasional outbreaks of virulent-systemic VSFCV infections, characterized by cutaneous edema, ulcerations of the head and feet, and occasionally jaundice and even death have been described in the USA and Europe and have also been observed in Switzerland during recent years and also in different region, Heilongjiang and Shanghai[18] of China.

Because of the limited knowledge of FCV genetics and variation, the isolation and identification of new isolates and the analysis of the FCV genome sequence is of particular significance.

Here we report the isolation and sequence analysis of a recombinant FCV isolate qd/2019/china, which was isolated from oropharyngeal swab of a dead domestic cat that exhibited seriously mouth and tongue ulceration.

\section{Materials And Methods}

\subsection{Clinical case}

In October 2019, a dead domestic cat Oropharyngeal swab was submitted for laboratory investigation. The apparent clinical symptom was thin and eye and nose was covered with purulent discharge, severe tongue damage and the whole tongue layer falls off, ulceration of the hard palate were also found. Necropsy of the cat showed hemorrhagic enteritis and lung Edema. The Oropharyngeal swab were collected and stored at $-80^{\circ} \mathrm{C}$ until the isolation of the FCV.

\subsection{Isolation and purification of the Virus.}

The protocol for Virus isolation and purification has been described previously. Brifely, CRFK cells (ATCC) were grown in DMEM(Gibco) containing 10\% fetal bovine serum (FBS) and 1\% penicillin-streptomycin $\triangle \mathrm{GIBCO}$. The cells were incubated at $37^{\circ} \mathrm{C}$ in a $5 \% \mathrm{CO} 2$ humidified cabinet. The collected Oropharyngeal swab were treated with $0.22 \mathrm{um}$ filter and inoculated into the CRFK monolayer, then the cytopathic effect 
(CPE) was observed at P1. When the CPE was obvious, the cultures were freeze-thawed and then harvested and stored at $-80^{\circ} \mathrm{C}$. The virus stocks were purified by plaque assay for three times.

\subsection{Identification of the Virus.}

The viral RNA was extracted from the cell culture supernatant using an TIANamp Virus DNA/RNA Kit (Tiangen Biotech, China) according to the manufacturer's protocol, and the CDNA was synthesized with random primer from the mRNA with a cDNA synthesis kit (TaKaRa, Tokyo, Japan), which was used for the template of PCR identification. the PCR primer was synthesised according a report[19], FCVF :GTAAAAGAAATTTGAGACAAT,FCV-R囚TACTGAAGWTCGCGYCT. The virus isolate were further identified by indirect immunofluorescence assay (IFA) using a cat polyclonal antibody against FCV[20].

\section{Genome cloning and sequencing}

The genome of the FCV strain qd/2019/china was cloned using a LA Taq PREMIX (LA Taq ${ }^{\text {TM }}$ Version 2.0,takara) $\bigotimes$ the primer were obtained based on conserved regions by Multiple sequence alignment analysis of several China FCV isolate(Table 1). The 5'- and 3'-end sequence was obtained by using the $5^{\prime}$ and 3' rapid amplification of cDNA ends (5'and3'RACE) kit (Invitrogen). The PCR products were cloned into the pMD18-T vector, and positive clones were used for sequencing and BLAST analyses were conducted to identify the related reference viruses of the qd/2019/china strain with the differential part of the genome and complete genome. BLAST analyses (http://blast.ncbi.nlm.nih.gov/Blast.cgi) were performed to identify the related reference viruses used in the following study of the qd/2019/china strain.

\section{Sequence analysis}

Capsid gene, VP1, is the most variable gene in the FCV genome, and phylogenetic analysis is usually undertaken based on alignment of the VP1 amino acid sequence. The sequence similarity was analyzed by DNAstar. Phylogenetic analysis of the full length FCV genome and capsid gene was performed using MEGA6.0. The bootstrap values were calculated according to 1000 replicates of the alignment.

\section{Recombination detection}

From the blast analysis, we found that different part of the $\mathrm{qd} / 2019 /$ china genome can result in different search results, which suggest the possibility of recombination of different isolate. Then to detect recombination signals in the FCV genomes $₫$ similarity comparisons and phylogenetic analyses Of the fulllength and part of FCV strain alignments were performed. The sequence used were download from NCBI. And Simplot 3.5.1 program were used in this study to verify recombination.

\section{Results}

\subsection{Virus isolation and identification}


The PCR assay was carried out to identify the FCV. The results showed that FCV was positive. Then the mouth swab was inoculated into the CRFK cells. The cytopathic effect (CPE) could be easily observed at $24 \mathrm{~h}$ post infection (Fig. 1).

After three rounds plaque purification, the CPE still stablely appeared on CRFK cells (Fig. 1A). FCV was still positive with PCR detection. Besides, qd/2019/china isotate was further identified in the infected CRFK cells using a cat polyclonal antibody against FCV(previous identified by our lab) (Fig. 1C). In this study, one FCV strain qd/2019/china was isolated from the mouth swab and was purified with plaque assay method.

\section{2 viral capsid amino acid sequence analysis}

Excitingly $\otimes T h e$ genome of the $q d / 2019 /$ china strain has been successfully sequenced. Then as for the capsid protein is significantly important for the study of FCV evolution and Immune escape. The sequence of the capsid amino acid were firstly analyzed. $64 \mathrm{FCV}$ isolate capsid protein sequence were retrieved from $\mathrm{NCBI}$, and DNAstar were used to analysis the sequence identity, Comparative pairwise analysis of the complete genome sequence of qd/2019/china VP1 with 64 VP1 sequence were conducted. FCV isolates showed sequence identity ranging from 83.9\% (ym3/2001/jp) to $91.1 \%$ (CH-JL4) with qd/2019/china, which were consistent with the previously reported genetically different FCV strains.

To further investigate the evolution of the qd/2019/china strain, a VP1 amino acid sequence phylogenetic tree was constructed using MEGA6. The evolutionary history was inferred using the maximum-likelihood $(\mathrm{ML})$ method $\triangle T$ The percentage of replicate trees in which the associated taxa clustered together in the

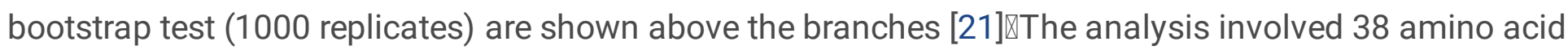
sequences. From the Evolutionary tree we can see that the qd/2019/china was on the same cluster with $\mathrm{CH}-\mathrm{JL} 4$, this is consistent with VP1 sequence analysis and complete genome BLUST analysis. qd/2019/china, CH-JL4, F65, UTCVM-H2, GX01-13, SH2014, UTCVM-H1 was in the same higher cluster.

Since FCV CH-JL4 challenge experiment on kittens indicate that it could lead to typical clinical symptoms $₫$ which means it had a stronger virulence. Here qd/2019/china was also isolated from a dead cat, it may also be a virulent strain, which need systematic virus challenge experiment and further investigation to prove.

\subsection{Complete genome sequence analysis}

To further understand the genetic origin of the $\mathrm{qd} / 2019 /$ china囚complete genome phylogenetic analysis were conducted using MEGA6هfigure 3). The FCV qd/2019/china strain was closely related to the $\mathrm{CH}-\mathrm{JL} 4$ sequence, and was in the same cluster with CH-JL4. besides, other China isolates (SH, XH, WZ-1, HRB-SS, GX01-13) , UTCVM-H2 and FCV/DD/2016/GE were in higher level cluster with qd/2019/china. The Chinese isolates were segregated into major genetic groups in the phylogenetic trees, and they were designated genogroups I and II. qd/2019/china ,CH-JL4, SH, XH, WZ-1, HRB-SS, GX01-13, UTCVM-H2 and FCV/DD/2016/GE all belong to the genogroups I. 


\subsection{Recombination analysis}

The BLUST result shown that the different part of the FCV genome show highest similarity with different FCV isolate. Given the potential significance of genetic recombination during evolutionary of picornaviruses[22], the recombination analysis were conduct with SimPlot. The results provided strong statistical support for a FCV qd/2019/china recombination event, The SimPlot graph clearly revealed the breakpoints that separated the genome of $\mathrm{qd} / 2019 /$ china into three regions, of which two fragments arose from CH-JL4 (regions 1-3821 nt and 5301-7707 nt) and one fragment originated from HRBSS(region 3821-5301 nt). The 3821-5301nt was the part of protease-polymerase(PP) gene based on the FCV CH-JL4.

To further validate the detected recombination signals, Phylogenetic tree of recombination region (the corresponding loci in the genome of qd/2019/china(3821-5301 nt) were constructed, which contain the genogroups I member(CH-JL4, SH, XH, WZ-1, HRB-SS, GX01-13, UTCVM-H2 and FCV/DD/2016/GE), as shown in the tree, $\mathrm{qd} / 2019 /$ china was in the same cluster with HRB-SS.

\section{Discussion}

FCV is one of the most common pathogen of domestic cats[23], which can cause severely respiratory and oral diseases(stomatitis and and gingivitis), although more and more cats were vaccinated, there are still many virus strain were isolated in china[1,23-25] . The FCV is single positive-strand RNA virus, which has high genomic variability for their RNA-dependent RNA polymerase( RdRP) Low-Fidelity . The detailed mechanisms of the emergence of new, genetically distinct FCV strains remain unknown[23].

In this study, a FCV qd/2019/china was isolated from a dead cat. This is the first FCV isolate of China Qingdao, Eastern coastal areas of China. The sequence analysis of capsid protein VP1 indicate that qd/2019/china have highest similarity with CH-JL4 (84.5\%).

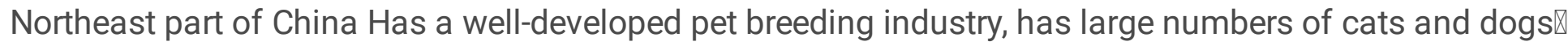
and many cats and dogs were transported to other parts of the country $\llbracket$ which promote the spread of the virus $₫$ the $\mathrm{qd} / 2019 /$ china may come from Jilin $₫$ which means transporting cats across regions may be a potential way to promote virus transmission and evolution.

As we all know, the recombination of virus is a common mechanism of virus evolution, existence of a recombinant FCV circulating within a naturally infected population of cats have been reported [26].Quasispecies represents as an effective adaptive strategy for the virus in an extremely heterogeneous viral population, which will evolve with better replicative capacity. but the FCV virus recombination across different regions have never been reported.

Here, the qd/2019/china may be a recombinate of HRB-SS(from Harbin) and CH-JL4(from Jilin). Now there was not any liscenced Live attenuated feline calicivirus vaccine in China, but the non-liscenced live attenuated FCV vaccine can not be absolutely prohibited, as some Clinical veterinarian thought the live 
attenuated vaccine is better than the killed vaccine. Here as for the evidence that FCV virus can recombines between different virus, the live vaccine virus can also be a potential recombination strains, the live FCV vaccine registration should be more rigorous.

FCV shows higher evolutionary rates than other viruses, and approximately $1.3 \times 10^{-2}$ to $2.6 \times 10^{-2}$ substitutions per nucleotide occur per year in the variable regions of the FCV capsid protein[27]Thus, the high genetic plasticity of the virus has led to the emergence of variants[28] ,PP protein is the RNAdependent RNA polymerase (RdRp) encoded by FCV and is indispensable for virus genomic replication[29], and PP and its precursor protein also have cysteine proteinase activity and are responsible for the proteolytic processing, which cut the ORF1 encoded large nonstructural proteins into five nonstructural proteins, p5.6, p32, p39, p30, VPg, PP. The PP proteins of FCV 2280 and F9 share 94\% amino acid identity. But strain 2280 grow faster than strain $\mathrm{F} 9$, and challenge experiments in cats showed that strain 2280 is more virulent than strain F9. PP is one of the most important nonstructural protein of FCV, N-Terminal Domain of PP can inhibit the host cell protein transcription[30], recently we found that PP can also effectively reduce the mRNA expression by promoting the degradation of host mRNAs[31].

In this study, we find that the region(3821-5301 nt) of qd/2019/china was recombinated from HRB-SS, and the region(3821-5301 nt) just belongs to pp region, the pp is a crucial protein to ensure the fidelity of RNA replication, the replace or change of PP will greatly change the virus characteristics, which may promote the evolution of FCV and promote the emergency of new FCV isolate. Besides, recombination has been associated with the expansion of viral host range[32], as many FCV isolate were isolated from Non-cat feline[1].

\section{Conclusions}

In this study a FCV strain qd/2019/china from China Qingdao was isolated. Phylogenetic analysis of the FCV genome reveal that it has the highest similarity with $\mathrm{CH}-\mathrm{JL} 4$, recombination analysis reveals that it maybe a recombinate virus of $\mathrm{CH}-\mathrm{JL} 4$ and HRB-SS. Besides, the recombination area belongs to PP region. Our results provide new evidence for the virus recombination contributing to FCV evolution.

\section{Declarations}

Acknowledgements: This work was funded by the Natural Science Foundation Of Hebei province『grant NO. C2021402005区, Science and Technology Research Project of Hebei Colleges and Universities囚grant NO. ZC2021214凹

Conflicts of Interest: The authors declare no financial or commercial conflicts of interest.

\section{Author contributions:}

Yongxiang Liu: Conceptualization, Methodology, Writing- Reviewing and Editing, 
Xiaoliang HU: Methodology, Reviewing and Editing

Lide Qin: Conceptualization,

\section{References}

1. Tian, J., et al., Molecular characterization of a feline calicivirus isolated from tiger and its pathogenesis in cats. Vet Microbiol, 2016. 192: p. 110-117.

2. Evermann, J.F., et al., Biological and pathological consequences of feline infectious peritonitis virus infection in the cheetah. Arch Virol, 1988. 102(3-4): p. 155-71.

3. Kadoi, K., et al., A strain of calicivirus isolated from lions with vesicular lesions on tongue and snout. New Microbiol, 1997. 20(2): p. 141-8.

4. Martella, V., et al., Analysis of the capsid protein gene of a feline-like calicivirus isolated from a dog. Vet Microbiol, 2002. 85(4): p. 315-22.

5. Di Martino, B., et al., Characterization of a strain of feline calicivirus isolated from a dog faecal sample. Vet Microbiol, 2009. 139(1-2): p. 52-7.

6. Martella, V., et al., Detection and Full-Length Genome Characterization of Novel Canine Vesiviruses. Emerg Infect Dis, 2015. 21(8): p. 1433-6.

7. Battilani, M., et al., Virulent feline calicivirus disease in a shelter in Italy: a case description. Res Vet Sci, 2013. 95(1): p. 283-90.

8. Rohayem, J., et al., Antiviral strategies to control calicivirus infections. Antiviral Res, 2010. 87(2): p. $162-78$.

9. Fino, V.R. and K.E. Kniel, UV light inactivation of hepatitis A virus, Aichi virus, and feline calicivirus on strawberries, green onions, and lettuce. J Food Prot, 2008. 71(5): p. 908-13.

10. Prikhodko, V.G., et al., Genetic characterization of feline calicivirus strains associated with varying disease manifestations during an outbreak season in Missouri (1995-1996). Virus Genes, 2014. 48(1): p. 96-110.

11. Domingo, E., et al., Basic concepts in RNA virus evolution. FASEB J, 1996. 10(8): p. 859-64.

12. Chen, S., et al., Molecular characterization of HLJ-073, a recombinant canine coronavirus strain from China with an ORF3abc deletion. Arch Virol, 2019. 164(8): p. 2159-2164.

13. Decaro, N., et al., Recombinant canine coronaviruses related to transmissible gastroenteritis virus of Swine are circulating in dogs. J Virol, 2009. 83(3): p. 1532-7. 
14. HM, W., et al., A natural recombinant PRRSV between HP-PRRSV JXA1-like and NADC30-like strains. Transboundary and emerging diseases, 2018. 65(4): p. 1078-1086.

15. Liu, D., et al., Recombination analyses between two strains of porcine reproductive and respiratory syndrome virus in vivo. Virus research, 2011. 155(2): p. 473-486.

16. He, C.Q., et al., The matrix segment of the" Spanish flu" virus originated from intragenic recombination between avian and human influenza $A$ viruses. Transboundary and emerging diseases, 2019.

17. Hu, C.J., et al., Nanoparticulate vacuolar ATPase blocker exhibits potent host-targeted antiviral activity against feline coronavirus. Sci Rep, 2017. 7(1): p. 13043.

18. Guo, H., et al., Isolation and molecular characterization of a virulent systemic feline calicivirus isolated in China. Infect Genet Evol, 2018. 65: p. 425-429.

19. Meli, M.L., et al., Molecular detection of feline calicivirus in clinical samples: A study comparing its detection by RT-qPCR directly from swabs and after virus isolation. J Virol Methods, 2018. 251: p. 5460 .

20. Wu, H., et al., Antiviral effect of lithium chloride on feline calicivirus in vitro. 2015. 160(12): p. 2935-2943.

21. Felsenstein, J., Confidence Limits on Phylogenies: An Approach Using the Bootstrap. Evolution, 1985. 39(4): p. 783-791.

22. Lukashev, A.N.J.R.i.M.V., Recombination among picornaviruses. 2010. 20(5): p. 327-337.

23. Zhao, Y., et al., Isolation and phylogenetic analysis of three feline calicivirus strains from domestic cats in Jilin Province, China. Arch Virol, 2017. 162(9): p. 2579-2589.

24. Liu, C., et al., Complete Genome Sequence of Feline Calicivirus Strain HRB-SS from a Cat in Heilongjiang Province, Northeastern China. 2014. 2(5): p. e00698-14-e00698-14.

25. Huimin, et al., Isolation and molecular characterization of a virulent systemic feline calicivirus isolated in China. 2018.

26. Coyne, K., et al., Recombination of Feline calicivirus within an endemically infected cat colony. 2006. 87: p. 921-926.

27. Coyne, K.P., et al., Evolutionary Mechanisms of Persistence and Diversification of a Calicivirus within Endemically Infected Natural Host Populations. 2007. 81(4): p. 1961-1971.

28. Coyne, K.P., et al., Large-Scale Spatial and Temporal Genetic Diversity of Feline Calicivirus. 2012. 86(20): p. 11356-67. 
29. Wei, L., et al., Proteinase-polymerase precursor as the active form of feline calicivirus RNAdependent RNA polymerase. J Virol, 2001. 75(3): p. 1211-9.

30. Wu, H., et al., N-Terminal Domain of Feline Calicivirus (FCV) Proteinase-Polymerase Contributes to the Inhibition of Host Cell Transcription. 2016. 8(7): p. 199.

31. Wu, H., et al., Feline Calicivirus Proteinase-Polymerase Degrades mRNAs to Inhibit Host Gene Expression. 2021.

32. E, S.-L. and H. EC, Why do RNA viruses recombine? Nature reviews. Microbiology, 2011. 9(8): p. $617-26$.

\section{Table}

Table 1 Primer used in the genome cloning

\begin{tabular}{|l|l|l|}
\hline Primer & Sequence $45^{\prime}$-3' 1 P & Position $^{\text {a }}$ \\
\hline FCV-TY-20F & GTAAAAGAAATTTGAGACAA & $1-20$ \\
\hline FCV-TY-2440-R & ATGTTGATTGGCGGGTAGTTC & $2440-2420$ \\
\hline FCV-TY-2420-F & GAACTACCCGCCAATCAACAT & $2420-2440$ \\
\hline FCV-TY-5333-R & TTAGCGCAGGTTGAGCACAT & $5333-5314$ \\
\hline FCV-TY-5314-F & ATGTGCTCAACCTGCGCTAA & $5314-5333$ \\
\hline FCV-TY-7709-R & CCCTGGGGTTAGGCGCGA & $7692-7709$ \\
\hline
\end{tabular}

a The position of primers was related to that in FCV strain CH-JL4 (Genbank accession: KT206207.1)

\section{Figures}



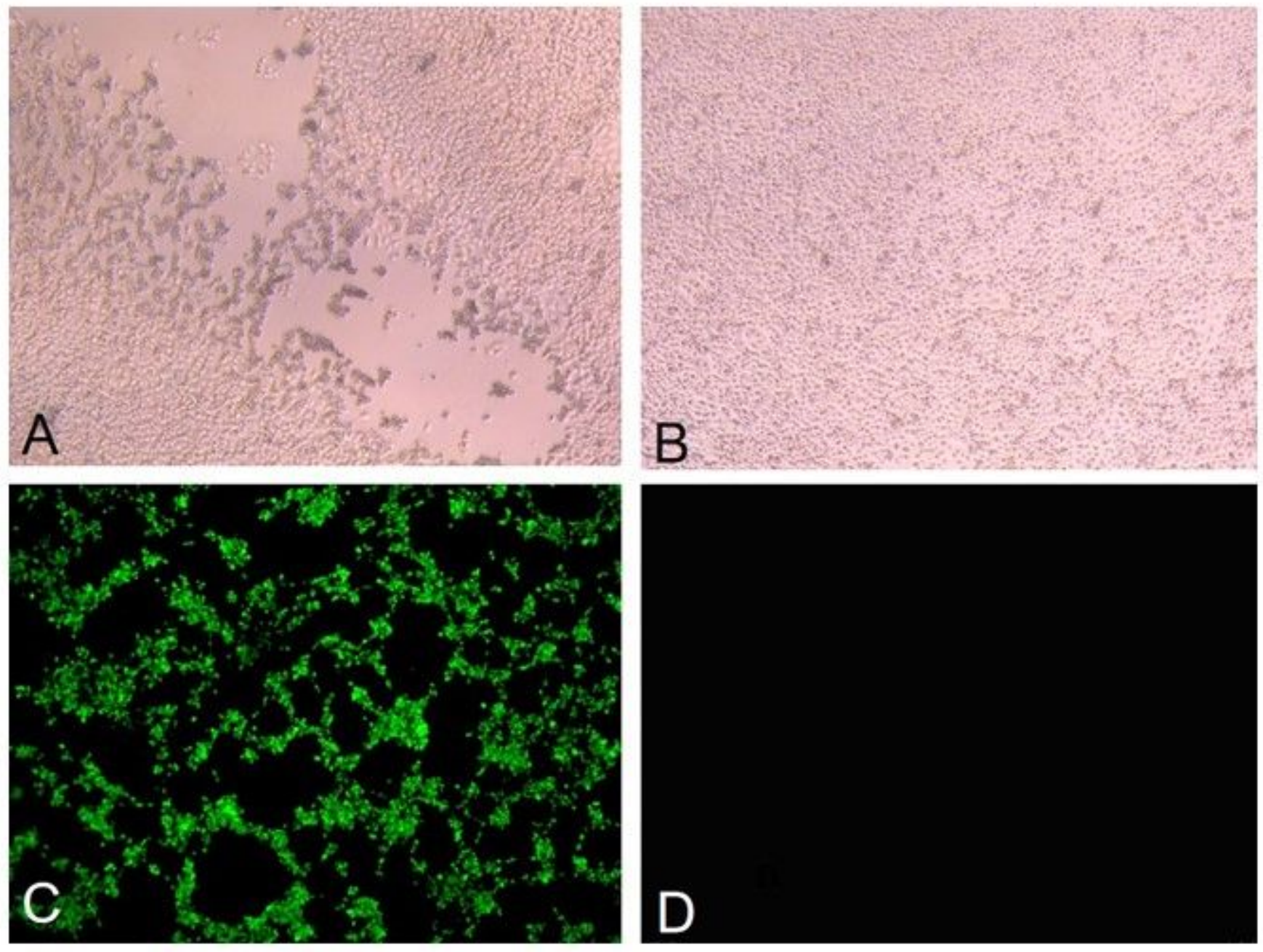

\section{Figure 1}

Virus isolation and identification . (A)Oropharyngeal swab was inoculated in CRFK cells. Typical CPE, cell aggregation, Rounding, falling off, enhanced shading were found. (B)While the physiological saline inoculated CRFK showed no CPE. (C) Bright green fluorescence were found in F81 cells infecting FCV. (D) Green fluorescence were not found in normal CRFK cells. 


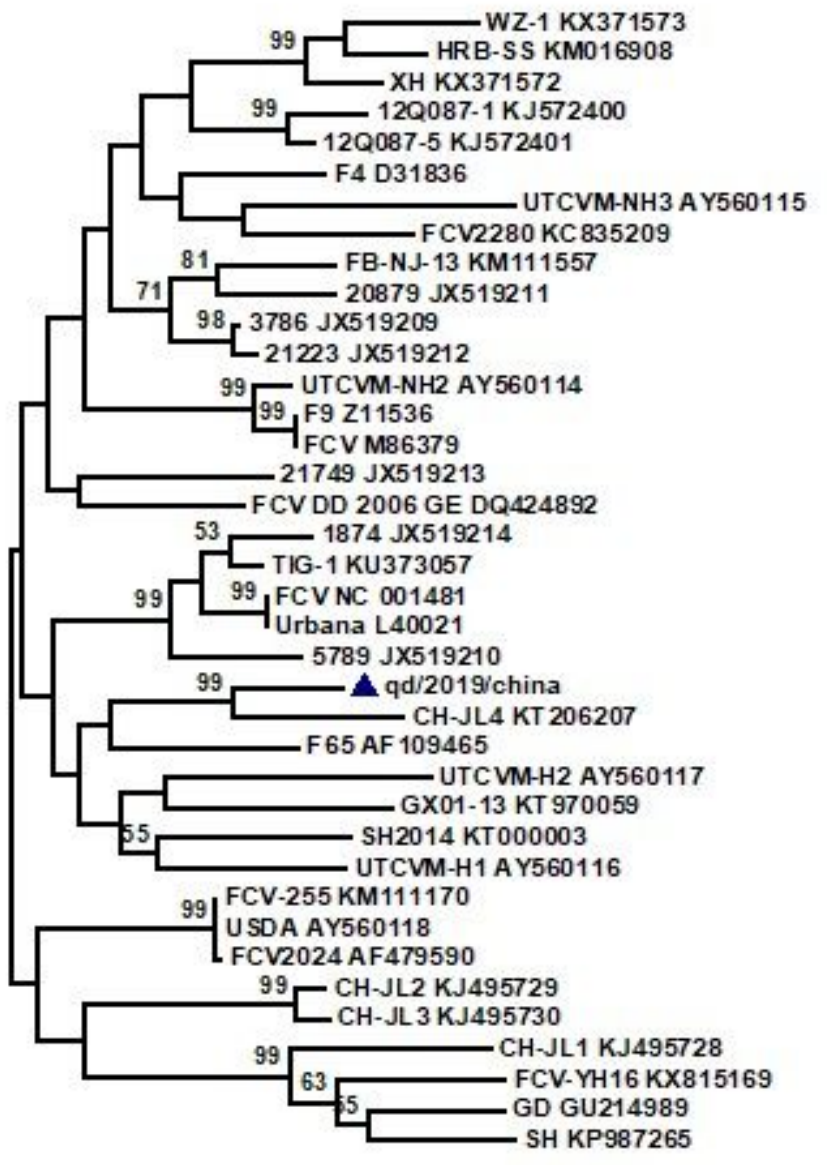

\section{Figure 2}

Phylogenetic tree of feline calicivirus (FCV) VP1 based on the amino acid sequence. The $\mathrm{qd} / 2019 /$ china was in the same cluster with $\mathrm{CH}-\mathrm{JL} 4$. The phylogenetic tree were constructed using the maximumlikelihood (ML) method based on the JTT matrix-based mode. 1000 bootstrap repetitions were performed for each analysis. Positions that contained gaps and/or missing data were eliminated. Bootstrap values $>50 \%$ are indicated. 


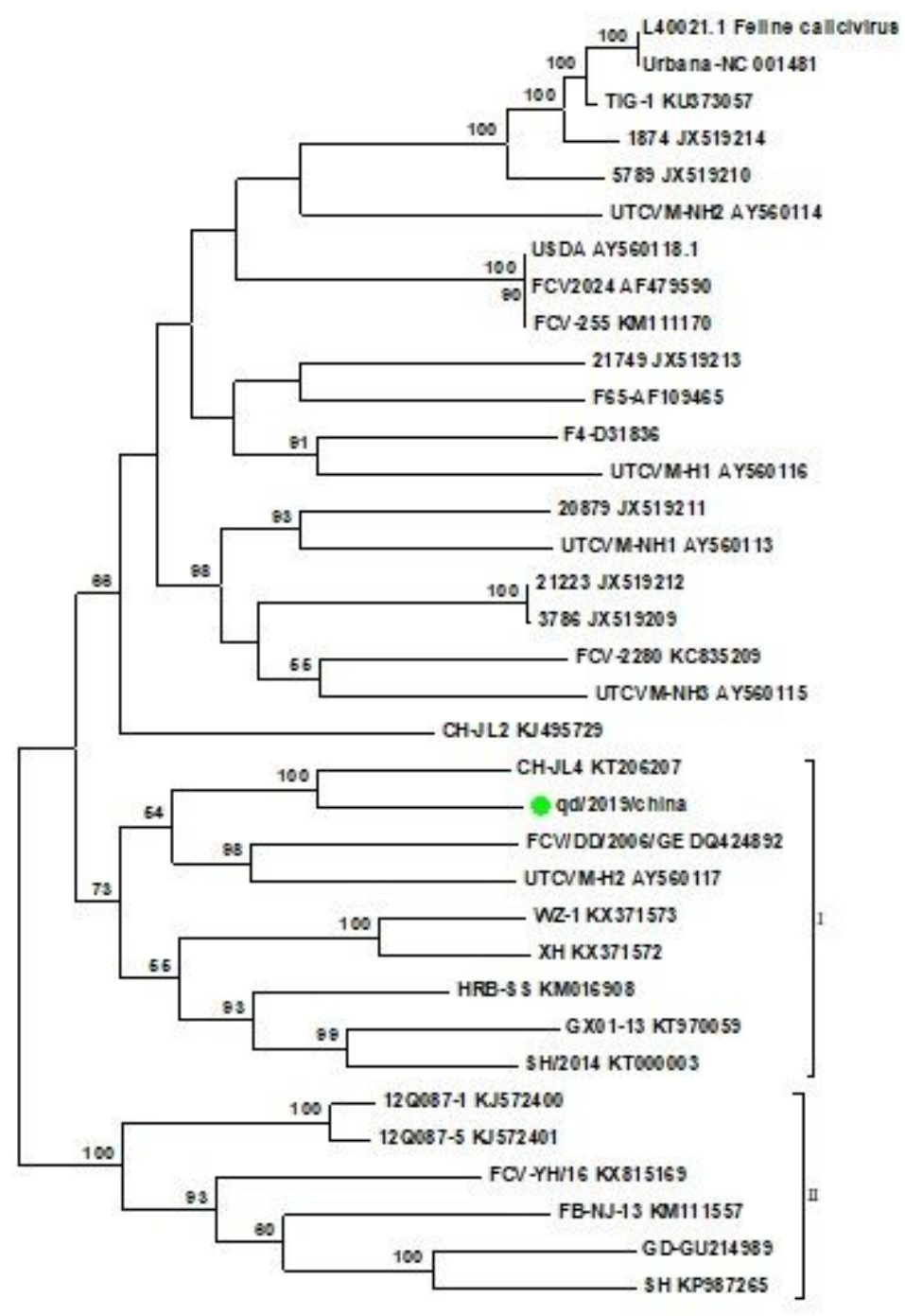

Figure 3

Phylogenetic trees of feline calicivirus complete genome. The phylogenetic tree was constructed using the neighbor-joining method with 1,000 bootstrap replicates and Kimura 2-parameter model in the MEGA 6.0 software package. qd/2019/china is labeled with a green circle. GenBank accession numbers are indicated on the branches. Bootstrap values $>50 \%$ are indicated. 


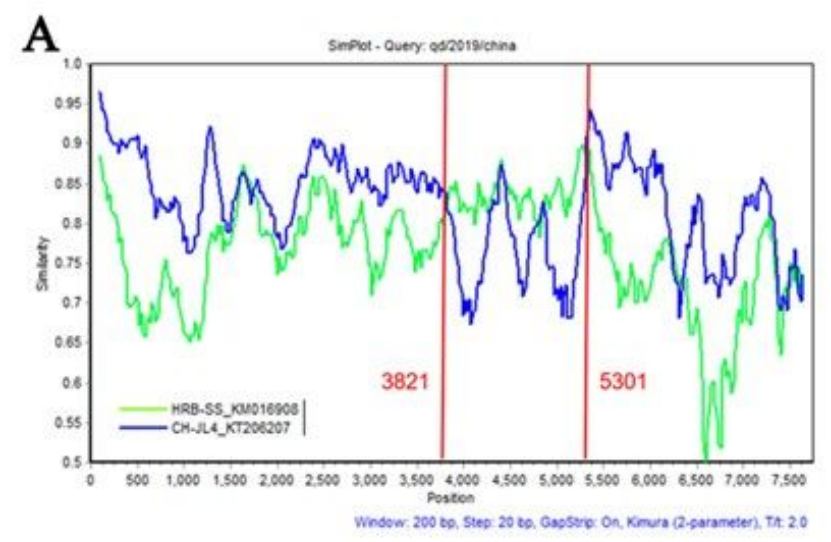

B

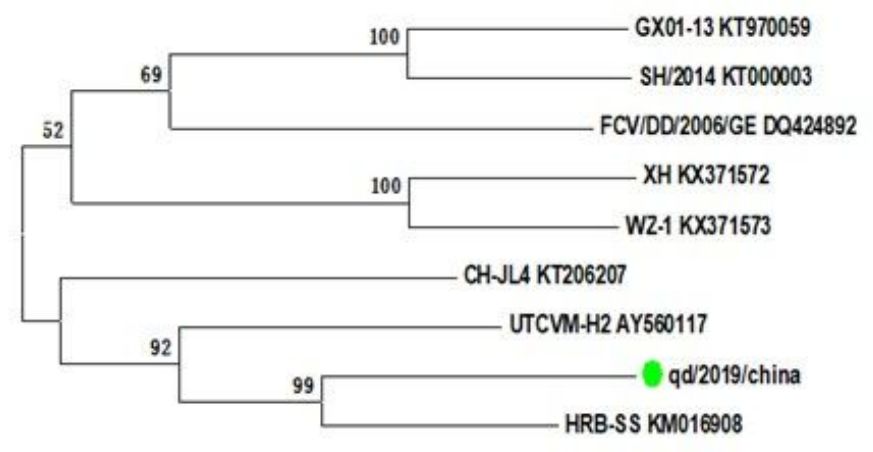

Figure 4

recombination analyses of the whole-genome nucleotide sequence of qd/2019/china. A. Recombination analysis based on the whole genome of qd/2019/china. Reference strains, HRB-SS(green) and $\mathrm{CH}-$ JL4(blue), were used as putative parental strains. The X-axis indicates the location of the query sequence, and the $\mathrm{Y}$-axis indicates the percentage of identity. B. Phylogenetic analysis based on genome region 3821-5301 nt. The phylogenetic trees were constructed using the MEGA6 software and the maximum likelihood algorithm, with 1000 bootstrap replications, and the Kimura 2-parameter substitution model.

\section{Supplementary Files}

This is a list of supplementary files associated with this preprint. Click to download.

- qd2019china.seq 